\title{
ÉTUDE DU STOCKAGE DES RÉSERVES PAR LES ABEILLES AU MOYEN D'UN SIROP DE SUCRE MARQUÉ AU ${ }^{141}$ Ce
}

\author{
Untersuchung der Lagerung von Futterreserven \\ durch die Bienen mittels Zuckerwasser, das \\ mit ${ }^{141}$ Ce markiert wurde
}

\author{
Ph. Douault, J. LouveauX, J. TheurkaufF, \\ P. AZGEUF * et J. PINTENA * \\ Station de Recherches sur l'Abeille et les Insectes Sociaux, I.N.R.A., \\ 91440 Bures-sur-Yvette \\ * Commissariat à l'Energie atomique, \\ Département des radio-êléments, B.P. No 2,91190 Gif-sur-Yvette
}

\author{
SUMMARY \\ STUDY OF THE HONEY STORAGE BY BEES BY MEANS \\ OF A SUGAR SYRUP TRAGED WITH ${ }^{141} \mathrm{Ce}$
}

\begin{abstract}
${ }^{141} \mathrm{Ce}$ (period of 32,5 days) was used to trace a syrup in the proportion of $500 \mu \mathrm{Ci} / 1$, which was supplied as feeding to seven honeybee colonies at the rate of one liter per week between the 23 rd of March and the 13 th of April 1973.

Dadant hives were used and supers were added on the $19^{\text {th }}$ of April at the beginning of an important rape nectar flow. Residual radioactivity in these supers was measured at the end of the nectar flow on the $29^{\text {th }}$ of May.

In each frame of all experimental hives, the presence of ${ }^{141} \mathrm{Ce}$ in honey stores was obvious. Nevertheless, the radioactivity rate was not the same in every frame, for some were more active than others. At the end of the experiment the radioactivity found in supers varied between 2,4 and $6,3 \%$ of the theoretical residual radioactivity. It corresponded to the moving by bees of 2,0 to $8,4 \mathrm{~g}$ of saccharose into every $\mathrm{kg}$ of gathered honey.

The theoretical and pratical meaning of bees moving feeding sugar into supers is discussed, with special regard to the risk of honey pollution by medicinal stuffs.
\end{abstract}




\section{RÉSUMÉ}

Le ${ }^{14} \mathrm{Ce}$ (période de 32,5 jours) a été utilisé pour marquer à la dose de $500 \mu \mathrm{Ci}$ par litre un sirop de sucre qui a été donné en nourrissement à 7 colonies d'abeilles à raison de 1 litre par semaine entre le 23 mars et le 13 avril 1973.

Les ruches utilisées étant du modèle Dadant, les hausses ont été posées le 19 avril au début d'une importante miellée de colza. La radio-activité résiduelle dans ces hausses a été mesurée le 29 mai, à la fin de la miellée.

Dans tous les cadres de toutes les ruches expérimentales on a pu mettre en évidence la présence de ${ }^{14} \mathrm{Ce}$ dans les réserves de miel. Cependant, la radio-activité n'était pas répartie de façon uniforme certains cadres étant plus actifs que d'autres. La radio-activité retrouvée en fin d'expérience dans les hausses variait entre 2,4 et $6,3 \%$ de la radio-activité résiduelle théorique. Elle correspondait au passage de 2,0 à 8,4 grammes de saccharose dans chaque $\mathrm{kg}$ de miel récolté.

La signification théorique et pratique des remontées de sucre de nourrissement dans les hausses est discutée, notamment en ce qui concerne le risque de pollution du miel par des substances médicamenteuses.

\section{INTRODUCTION}

L'emploi de radio-isotopes pour marquer un sirop de sucre donné aux abeilles a déjà permis de réaliser de nombreuses expériences. Grâce à cette technique Nixon et Ribbands (1952) ont montré que la nourriture liquide fait l'objet dans la ruche de transferts continuels d'ouvrière à ouvrière. Courtors et Lecomte (1958) ont constaté, en marquant des abeilles avec de l'or 198, que la totalité de la population de la ruche devient radioactive en 36 heures. D'autres expériences (Gösswald et Kloft, 1958 - Courtors et al. 1961 - Douault, 1967) ont permis de confirmer la rapidité de la diffusion de l'élément marqué dans la ruche et de préciser les modalités de cette diffusion en fonction de différents facteurs.

Jusqu'ici les différents auteurs se sont surtout préoccupés de marquer des abeilles, le sirop ne constituant qu'un véhicule commode pour le radio-isotope. En fait, on dispose avec les radio-éléments, de traceurs permettant de suivre avec précision le cheminement de l'aliment lui-même ce qui est précieux pour l'étude de ses transformations physico-chimiques, de son stockage dans le rayon et de sa consommation. C'est pourquoi nous avons pensé intéressant de suivre sur quelques ruches soumises avant la miellée à un nourrissement spéculatif le cheminement du sirop distribué aux abeilles et son passage ultérieur éventuel dans les hausses lorsque celles-ci sont posées au début de la miellée.

A côté de l'intérêt scientifique d'une expérimentation permettant de savoir ce que devient le sirop de nourrissement il faut noter son intérêt pratique. Dans l'état actuel des techniques de production du miel, les nourrissements au sucre constituent une nécessité économique, qu'il s'agisse de sauver des colonies de la famine ou d'obtenir leur développement rapide ou encore d'administrer une drogue destinée à la lutte contre les maladies des abeilles. La conséquence 
de la généralisation des nourrissements au sucre est le risque indiscutable de voir passer dans le miel soit du sucre industriel, ce qui constitue une fraude, soit une substance médicamenteuse, ce qui pourrait éventuellement présenter un danger pour la santé du consommateur. Ce problème est connu depuis fort longtemps et il a déjà suscité de nombreux commentaires mais peu de recherches précises.

JAChimowicz (1964) a nourri au sucre en poudre de mai à juillet 6 colonies d'abeilles. Il a observé la consommation de ce sucre en rapport avec la miellée et avec les conditions météorologiques. Trois des ruches ont reçu du sucre en poudre coloré au bleu de méthylène. Les miels récoltés ont été analysés et la mesure de la coloration a permis d'estimer la quantité de sucre passée dans le miel. Celle-ci était négligeable et, de toute façon, impossible à mettre en évidence par les méthodes analytiques classiques.

Roussead et Tabarly (1962) ont recherché des antibiotiques présents dans la ruche après administration d'un sirop médicamenteux et ils ont pu effectivement les retrouver mais les conditions expérimentales de leurs travaux étaient assez éloignées de la pratique apicole.

D'une façon générale les caractères physico-chimiques des miels résultant d'un nourrissement massif au sucre ont fait l'objet de nombreux travaux qui ont montré que ces produits ont une composition qui s'écarte plus ou moins de celle du miel et qu'il est possible de les reconnaître dans la mesure où ils sont suffisamment purs (Hadorn et Zürcher, 1963 - Vorwohl, 1964). Par contre, on a très peu étudié ce qui se passe dans la pratique chez l'apiculteur respectueux des lois et qui ne nourrit pas les abeilles lorsque les hausses sont posées ou que la miellée a commencé.

Mettant à profit l'extrême sensibilité des méthodes de détection de la radio-activité nous nous sommes délibérément placés dans des conditions loyales et normales afin d'estimer le risque encouru par l'apiculteur sur le plan pénal et par le consommateur sur le plan de l'hygiène alimentaire du fait des nourrissements spéculatifs ou médicamenteux.

\section{MATÉRIEL ET MÉTHODES}

\section{1. - Le rucher expérimental}

Le rucher destiné à l'expérimentation a été constitué au début du mois de mars 1973. Huit ruches Dadant 10 cadres provenant de la Station de recherches sur l'abeilıe et les insectes sociaux ont été installées sur une parcelle entièrement clôturée située sur la commune de Magny-les-Hameaux (Yvelines). Ces ruches étaient peuplées d'abeilles de la race noire commune; toutes les reines étaient nées en 1972 et elles étaient scurs. La principale ressource mellifère du lieu était le colza (Brassica napus var. oleifera) cultivé à proximité du rucher sur plusieurs dizaines d'hectares. 
Deux lots de ruches ont été constitués par séparation des colonies largement approvisionnées en miel d'avec les plus légères. Le remplacement de quelques cadres a permis d'accentuer les différences de poids dans le sens souhaité. Le tableau 1 donne l'indication du poids des 7 ruches qui restaient le 23 mars après élimination d'une colonie orpheline.

Les nourrissements avec le sirop marqué ont commencé aussitôt après la pesée du 23 mars. Toutes les colonies étaient à ce moment en parfait état sanitaire et très populeuses. Les provisions de miel étaient normales pour la saison dans le lot des ruches lourdes et anormalement faibles dans le lot des ruches légères.

\section{Choix du radio-isotope}

L'expérimentation devant avoir lieu dans la nature et la détection devant être effectuée environ 2 mois après le premier nourrissement, il importait de choisir un radio-isotope présentant des caractéristiques bien définies. Pour faciliter la détection et pour diminuer l'absorption du rayonnement par les matériaux de la ruche il fallait utiliser un émetteur $\gamma$. Pour que l'activité soit encore détectable au bout de 60 jours compte tenu de la limitation imposée au départ, il fallait que la période soit de l'ordre de 30 jours. Le radio-isotope dont les caractéristiques se rapprochent le plus de celles requises était le Cérium 1411 ( $\left.{ }^{141} \mathrm{Ce}\right)$.

- Période 32,5 jours

- Énergie $\beta 0,581 \mathrm{Mev}$

- Énergie $\gamma 0,145 \mathrm{Mev}$

- Activité spécifique supérieure à $100 \mathrm{mCi.g-1}$

Le ${ }^{141} \mathrm{Ce}$ est livré par le Centre d'étude nucléaires de Saclay sous forme de chlorure en solution dans l'acide chlorhydrique. Par décroissance il donne le praséodyme 141 ( ${ }^{141} \mathrm{Pr}$ ) qui est stable. Sa période relativement courte permet la décontamination par simple décroissance lorsque l'élimination de l'activité ne peut pas se faire par des moyens physiques ou chimiques. Le temps de décontamination est d'environ 10 périodes, soit 320 jours.

\section{3. - Calcul de l'activité nécessaire}

La dose de radio-isotope à incorporer à chaque nourrissement a été calculée en tenant compte de deux impératifs contradictoires, à savoir, la nécessité de conserver une radio-activité détectable au bout de deux périodes et l'obligation d'éviter la contamination de l'environnement conformément aux règles imposées par la Commission interministérielle d'utilisation des radioéléments. C'est en fonction de ces considérations qu'on a fixé à $500 \mu$ Ci par ruche l'activité apportée par chaque nourrissement, soit un total de $12 \mathrm{mCi}$ pour l'ensemble de l'expérimentation prévue pour 8 colonies.

En admettant que les abeilles rejettent d'une manière continue cette radio-activité dans la nature pendant une période de 8 mois et en tenant compte de la décroissance du ${ }^{141} \mathrm{Ce}$ dans les ruches pendant cette même période, le calcul montre que l'activité totale rejetée dans la nature pendant 8 mois est de $360 \mu \mathrm{Ci}$, ce qui reste dans les limites admissibles. Par ailleurs, l'activité choisie est suffisante pour permettre après deux périodes de décroissance de détecter $0,1 \%$ de l'activité initiale dans l'ensemble formé par les 9 cadres d'une hausse.

\section{4. - Toxicité chimique du cérium et toxicité radio-chimique du ${ }^{141} \mathrm{Ce}$ à l'égard des abeilles}

Le ${ }^{141}$ Ce est produit par réaction $(n, \gamma)$ à partir du ${ }^{140} \mathrm{Ce} q u i$ est stable. La solution de ${ }^{141} \mathrm{Ce}$ utilisée contient donc du cérium inactif d'une concentration de l'ordre du gramme par $100 \mathrm{mCi}$. Il s'ensuit que l'utilisation d'une telle solution pour le nourrissement des abeilles pourrait éventuellement conduire à leur intoxication chimique ou engendrer un effet radio-chimique. C'est pourquoi, avant toute expérience de marquage nous avons procédé à des essais de toxicité au laboratoire.

a) Toxicité du cérium inactif.

Le test de toxicité a été fait à partir d'une solution de chlorure cérique $\left(\mathrm{Ce} \mathrm{Ci}_{4}\right)$ en milieu faiblement chlorhydrique mélangée à du miel. Ce mélange a été donné en nourrissement à des abeilles maintenues à l'étuve à $30^{\circ} \mathrm{C}$ dans des cagettes de laboratoire. Chaque cagette contenait 
50 abeilles. Dans un premier lot de 3 cagettes on a ajouté le cérium à la nourriture à la dose de 6 ug par cagette. Dans un second lot on l'a ajouté à la dose de $12 \mu \mathrm{g}$. Un $3^{\mathrm{e}}$ lot a servi de témoin. La dose administrée a été calculée en considérant que, sur le terrain, les colonies recevraient en moyenne $5 \mathrm{mg}$ de cérium à chaque nourrissement. Au bout de 3 semaines la mortalité était la même dans les 3 lots de cagettes ce qui indique qu'à la dose utilisée le cérium n'est pas toxique pour les abeilles.

b) Effet radio-chimique du ${ }^{141} \mathrm{Ce}$.

L'abeille butineuse supporte sans dommage apparent une dose d'irradiation de 18000 Roentgens et on peut admettre que la période biologique du ${ }^{141} \mathrm{Ce}$, élément non métabolisable, est du même ordre de grandeur que celle du ${ }^{108} \mathrm{Au}$, soit 3 jours (Courtors et LeComte, 1958). Considérons une colonie de 40000 abeilles recevant 3 nourrissements ayant chacun une activité de $500 \mu \mathrm{Ci}$, soit un total de $1500 \mu \mathrm{Ci}$. En admettant qu'administrés en une seule fois ces $1500 \mu \mathrm{Ci}$ se répartissent uniformément entre tous les individus de la ruche, le calcul montre que, compte tenu de l'énergie $\beta$ du ${ }^{141} \mathrm{Ce}$ qui seule intervient pratiquement pour l'irradiation, chaque abeille pourrait recevoir une dose d'environ 30 Roentgens, laquelle est fort éloignée de la dose létale qui est de l'ordre de 90000 Roentgens.

\section{5. - Marquage et distribution du sirop}

Chacun des nourrissements a été marqué par $500 \mu \mathrm{Ci}$ de ${ }^{141} \mathrm{Ce}$. Les solutions ont été préparées de la façon suivante : à un litre de sirop contenant $600 \mathrm{~g}$ de sucre on ajoute $0,5 \mathrm{ml}$ d'une solution de ${ }^{141} \mathrm{Ce}$ d'activité volumique $1 \mathrm{mCi} \cdot \mathrm{ml}^{-1}$. Après homogénéisation à l'aide d'un agitateur magnétique, la solution est maintenue à $40^{\circ} \mathrm{C}$ jusqu'au moment de sa mise en place dans le nourrisseur à la partie supérieure de la ruche. Chaque nourrisseur est protégé du froid par une gaine isolante de façon à faciliter la prise de nourriture par les abeilles qui absorbent plus facilement le sirop tiède.

\section{6. - Calendrier des opérations de nourrissement, de pesées et de pose des hausses}

- 23 mars 1973 : Pesée des ruches (Tableau 1). Premier nourrissement avec un litre de sirop marqué par ruche.

TABL. 1. - Poids des ruches expérimentales au 23 mars avant le début des nourrissements

TAB. 1. - Gewicht der Versuchsbienenvölker am 23. März vor Beginn der Fütterung

\begin{tabular}{c|c|c|c}
\hline \multicolumn{2}{c|}{$\begin{array}{c}\text { Ruches légères } \\
\text { Leichte Bienenstöcke }\end{array}$} & \multicolumn{2}{|c}{$\begin{array}{c}\text { Ruches lourdes } \\
\text { Schwere Bienenstöcke }\end{array}$} \\
\hline No & Poids (kg) \\
Nr. & Gewicht (kg) & No & Poids (kg) \\
& & Nr. & \\
\hline 510 & 21 & 556 & 29 \\
528 & 20 & 571 & 29 \\
536 & 22 & 572 & 30 \\
& & 573 & 29,5 \\
\hline \hline
\end{tabular}

- 30 mars : second nourrissement : 1 litre de sirop par ruche. Ce sirop n'a pas été marqué. En effet, les mauvaises conditions atmosphériques du début du printemps ont rendu nécessaire un apport de sucre plus important que prévu mais, comme il convenait de respecter la dose totale de radio-isotope autorisée, le second nourrissement n'a pas été marqué.

- 6 avril : $3^{\mathrm{e}}$ nourrissement $: 1$ litre de sirop marqué par ruche. 
- 13 avril : $4^{\mathrm{e}}$ nourrissement $: 1$ litre de sirop marqué par ruche.

- 19 avril: pesée des ruches. Pose de la $1^{\text {re }}$ hausse.

- 17 mai : pesée des ruches. Pose de la $2^{e}$ hausse.

- 29 mai : dernière pesée des ruches. Enlèvement des hausses. Mesure de la radioactivité résiduelle dans les hausses.

Toutes les ruches n'ont pas absorbé la totalité du sirop marqué fourni. Les ruches $\mathrm{n}^{\text {os }} 571$, 556 et 573 n'ont pris que 3,5 litres au lieu de 4 . Le sirop non absorbé correspond au dernier nourrissement; il s'agit donc de sirop marqué et nous avons tenu compte de ce déchet dans les calculs.

\section{7. - Matériel et méthodes de détection de la radio-activité résiduelle}

L'ensemble de détection comprenait une échelle de mesure ECP 2 à alimentation autonome associée à un ensemble SPP 3 . L'échelle de comptage était reliée à une sonde SGSIN équipée d'un cristal $\mathrm{NaI}$ (iodure de sodium activé au thallium) de 1 pouce $3 / 4 \times 2$ pouces. La sonde était montée sur un dispositif à balayage permettant par 10 mesures successives de chacune 100 secondes d'intégrer toute l'activité contenue dans un cadre de hausse Dadant. La division d'un cadre est représentée par la figure 1, l'appareil de balayage par la figure 2.

La sonde étant placée en position 1, l'ensemble de comptage enregistre le nombre de coups dus à l'activité contenue dans cette fraction du cadre pendant 100 secondes. On passe à la position 2 en effectuant la même mesure et ainsi de suite jusqu'à la position 10. En faisant la somme des 10 taux de comptage obtenus après soustraction du bruit de fond de l'appareil on peut connaître avec précision l'activité contenue dans le cadre. On sait, grâce à un étalonnage préalable que pour $0,27 \mu \mathrm{Ci}$ de ${ }^{141} \mathrm{Ce}$ on obtient un taux de comptage de 27000 coups nets.

Notons que pour augmenter les taux de comptage et pour augmenter par conséquent la sensibilité de la méthode on a utilisé le SPP 3 en position “ intégrale », ce qui permet de compter, à partir d'un seuil bas qui élimine le bruit de fond électronique de l'appareil, les impulsions dues à la totalité du spectre énergétique du ${ }^{111} \mathrm{Ce}$. Cette technique de comptage, comparée à celle dite (c en différentiel ", c'est-à-dire dans la bande entourant le pic photoélectrique du ${ }^{141} \mathrm{Ce}$, obtenue par deux seuils préréglés de part et d'autre de ce pic, ne permet pas d'éliminer les impulsions dues à des retombées radioactives éventuelles contaminant le miel. Si on se reporte aux travaux de Racoveanu et al. (1965) les calculs montrent que cette interférence est négligeable et entraîne une erreur sur les résultats qui est très inférieure à l'erreur statistique des taux de comptage.

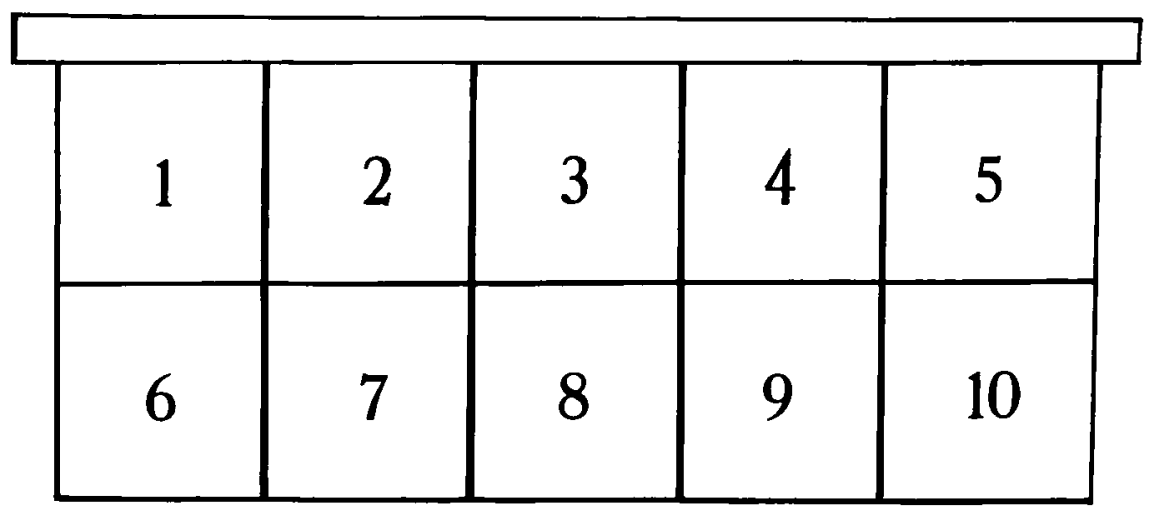

FIG. 1. - Schéma d'un cadre de hausse Dadant et répartition des champs de détection de la radio-activité (de 1 à 10).

Aв8. 1. - Schema einer Wabe im Dadant-Aufsatz mit der Verteilung der Felder zum Radioaktivitätsnachweis (1 bis 10). 

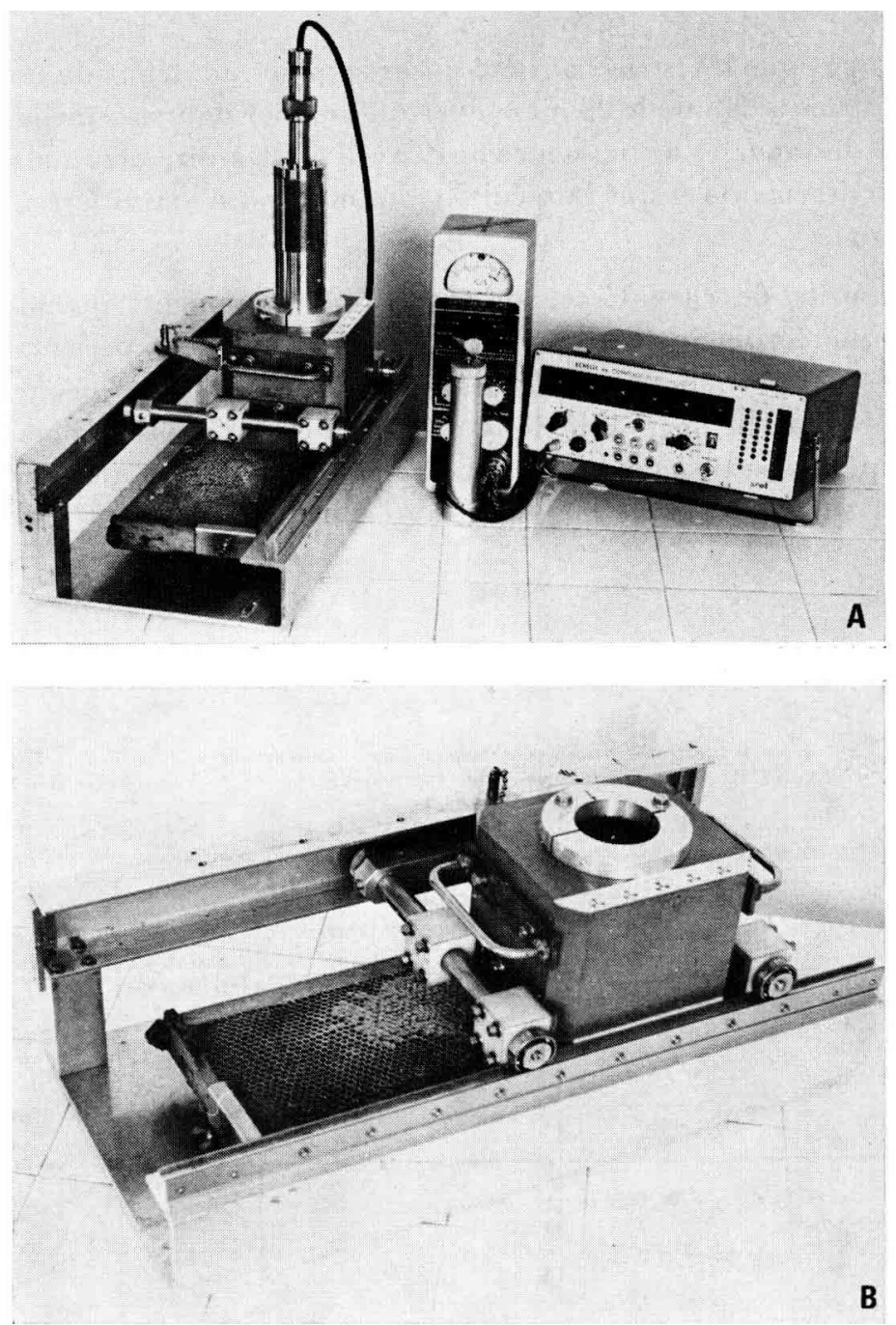

Fig. 2. - A - Vue d'ensemble du matériel de mesure de la radio-activité dans les cadres de hausse. De gauche à droite :

- chariot supportant la sonde $\%$.

- scintillomètre SPP 3.

- échelle de comptage ECP 2.

B - Détail du chariot porte-cadre

AвB. 2. -- A - Gesamtansicht der Vorrichlung zur Radioaktivitäts-Messung in den Waben des Aufsatzes. Von links nach rechts:

— Schlitten mit der Sonde $\%$.

- Szintillometer SPP 3.

- Skala ECP 2. 


\section{RÉSULTATS}

Rappelons que les ruches ont été mises en place au début du mois de mars, que l'expérience a débuté le 23 mars avec un premier nourrissement radio-actif, que la pose des hausses a commencé le 19 avril c'est-à-dire une semaine après la fin des nourrissements et que la récolte et les mesures d'activité résiduelle ont eu lieu le 29 mai.

Il est utile de considérer, au moins sommairement, quelles furent les conditions météorologiques entre le début et la fin de l'expérience de façon à comprendre l'évolution du poids des ruches. Nous utiliserons pour celà les relevés de la station météorologique d'Orly qui est l'une des plus proches du lieu des expériences et nous nous contenterons des données décadaires qui sont amplement suffisantes pour nos besoins (Tableau 2).

TaBL. 2. - Conditions météorologiques au cours de la période des essais (23 mars-29 Mai 1973) Relevés effectués à Orly (Bulletin décadaire d'observations quotidiennes. Direction de la Météorologie Nationale).

TAB. 2. - Witterungsverhältnisse während der Versuchszeit (23.3. - 29.5.73)

Wetterbericht von Orly. (ZehnTagebericht der täglichen Erhebungen. Direktion der nationalen Meteorologie)

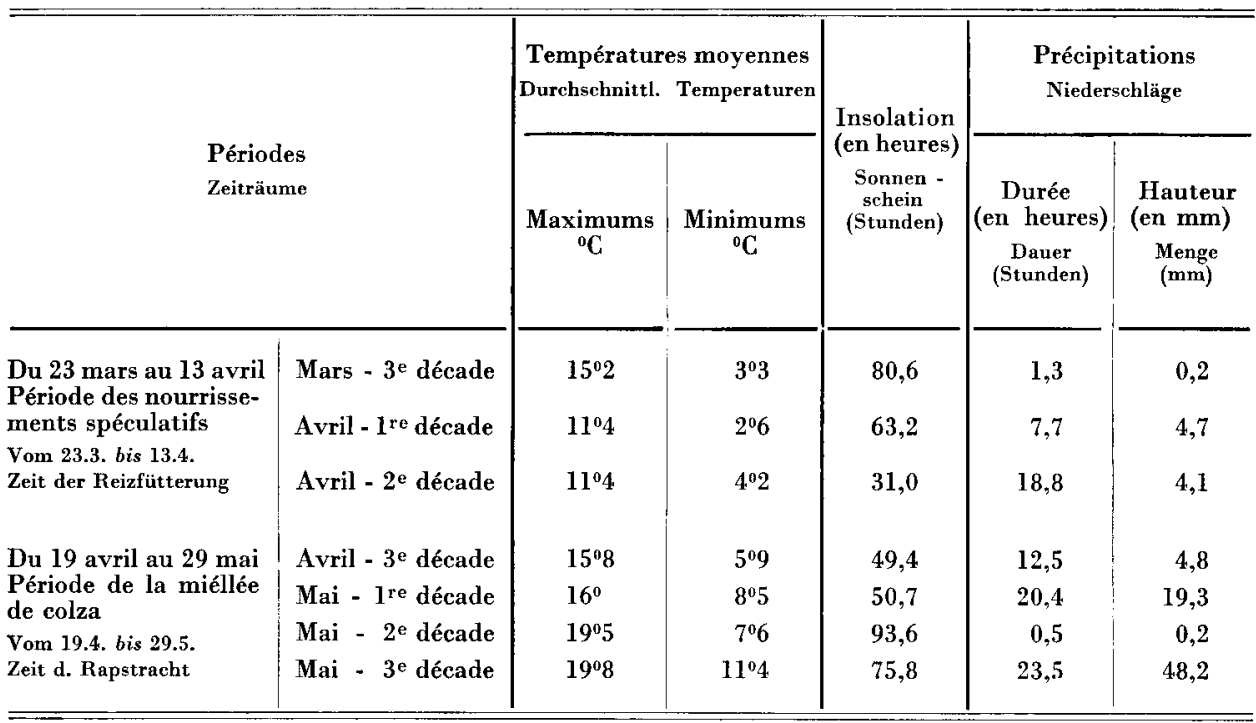

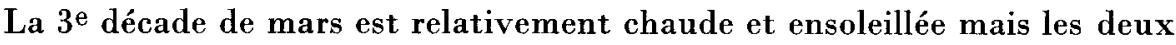
premières décades d'avril sont froides, humides et peu ensoleillées. Un temps plus doux revient dans la $3^{e}$ décade d'avril. Le mois de mai est relativement chaud et la $2^{\mathrm{e}}$ décade amène des conditions très favorables à la miellée de colza. 
L'examen des variations du poids des ruches entre le 23 mars et le 29 mai (Tableau 3) vient bien confirmer les données météorologiques. Du 23 mars au 19 avril le poids des ruches varie très peu, soit en plus soit en moins, malgré le nourrissement fourni aux colonies. On peut admettre que ce nourrissement couvre tout juste les besoins des ruches les plus légères. Il est moins bien absorbé par les ruches les plus lourdes puisque dans trois cas sur quatre il reste $1 / 2$ litre de sirop non consommé au moment de la pose des hausses.

TaBL. 3. - Poids et variations de poids des ruches expérimentales au cours de la période des essais

TAв. 3. - Gewicht und Gewichtsveränderungen der Versuchsvölker während der Versuchszeit

\begin{tabular}{|c|c|c|c|c|c|c|c|c|}
\hline & $\begin{array}{c}\text { Numéro des ruches } \\
\text { Nummer der Bienenstöcke }\end{array}$ & 510 & 536 & 528 & 572 & 571 & 556 & 573 \\
\hline 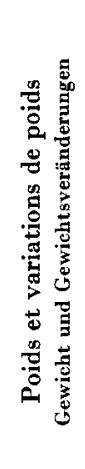 & $\begin{array}{l}\text { Poids des ruches au } 23 \text { mars }(\mathrm{kg}) \ldots \\
\text { Gewicht der Bienenstöcke am } 23.3 .(\mathrm{kg}) \\
\text { Volume du nourrissement (en l.) ... } \\
\text { Futtermenge (in 1) } \\
\text { Variation du poids des ruches entre } \\
\text { le } 23 \text { mars et le } 19 \text { avril }(\mathrm{kg}) \ldots \ldots \\
\text { Gewichtsveränderungen der } \ldots \text { Bienenstöcke } \\
\text { zwischen dem } 23.3 . \text { und 19.4. (kg) } \\
\text { Variation du poids des ruches entre } \\
\text { le } 19 \text { avril et le } 17 \text { mai }(\mathrm{kg}) \ldots \ldots \\
\text { desgl. zwischen dem } 19.4 . \text { n.17.5. }(\mathrm{kg}) \\
\text { Poids de la récolte le } 29 \text { mai }(\mathrm{kg}) \ldots \\
\text { Gewicht des geernteten Honigs am } 29.5 .(\mathrm{kg})\end{array}$ & +23 & $\begin{array}{r}+18 \\
19,3\end{array}$ & $0^{2}$ & +16 & $+14,5$ & $\begin{array}{r}+17,5 \\
22,9\end{array}$ & $\begin{array}{r}+12 \\
18\end{array}$ \\
\hline
\end{tabular}

Le 19 avril, l'amélioration du temps et l'évolution des floraisons de colza le permettant, il devient possible de poser les hausses et celles-ci se remplissent rapidement. En un mois le gain de poids est de 12 à $23 \mathrm{~kg}$ par ruche. Au cours de la dernière période, du 17 au 29 mai, la miellée se termine et on ne trouvera qu'une différence assez faible en général entre le poids du contenu des deux hausses le 19 mai et le gain de poids précédemment enregistré.

On peut considérer que la récolte de miel de colza faite au rucher de Magny-les-Hameaux en 1973 est très satisfaisante. D’une façon générale les premières hausses sont pleines; les secondes hausses contiennent toutes $\mathrm{du}$ miel mais en quantité variable. Les reines ont pondu légèrement dans la 1 re hausse mais la surface occupée par le couvain ne dépasse pas $5 \%$.

Examinons maintenant ce qu'est devenu le sirop marqué distribué (en quantités raisonnables) bien avant la pose des hausses et pendant une période où les besoins alimentaires des abeilles excédaient les rentrées de nectar. Deux hypothèses sont possibles. Dans la première on suppose que le sirop a été 
intégralement consommé ou stocké définitivement dans le corps de ruche auquel cas on n'en retrouvera pas trace dans les hausses. Dans la seconde on admet qu'une partie au moins du sirop peut ne pas avoir été consommée et que la pose des hausses entraîne pour les abeilles la possibilité de modifier l'agencement du nid avec comme conséquence le déplacement vers la partie supérieure des stocks de nourriture. Dans cette seconde hypothèse il doit être possible de retrouver dans les hausses une partie des $1500 \mu \mathrm{Ci}$ introduits dans le corps de ruche par les nourrissements marqués.

Étant donné la décroissance naturelle de la radio-activité du ${ }^{141} \mathrm{Ce}$ on a calculé tout d'abord la radio-activité résiduelle totale qu'il serait possible de retrouver dans le cas où tout le sirop marqué aurait été déposé dans les hausses sans pertes ni consommation. On a appliqué la formule de décroissance :

$$
\mathrm{A}=\mathrm{A}_{\mathrm{o}} \mathrm{e}^{-0,693} \frac{t}{32,5}
$$

dans laquelle $t$ est exprimé en jours et représente le temps écoulé entre la date du nourrissement et le 29 mai. On a tenu compte du volume de sirop effectivement consommé. Pour les colonies ayant absorbé 4 litres de sirop, cette radio-activité théorique maximum était de $466 \mu \mathrm{Ci}$ au 29 mai. Elle n'était à la même date que de $373 \mu \mathrm{Ci}$ pour les colonies ayant absorbé 3,5 litres de sirop.

Ayant procédé à la mesure cadre par cadre de la radio-activité résiduelle dans les 14 hausses utilisées on a recueilli des données figurant au tableau 4. A raison de 9 cadres par hausse, de 10 mesures par cadre et de 100 secondes par mesure, le travail a dû être étalé sur deux jours mais ce délai est trop court pour qu'il en résulte une diminution appréciable de la radio-activité entre le début et la fin des mesures.

L'examen du tableau 4 montre à l'évidence que c'est la seconde hypothèse qui doit être retenue. La radio-activité résiduelle n'est nulle que dans les cadres vides. Elle est la plus forte dans la $1^{\text {re }}$ hausse qui contient du miel operculé et un peu de couvain dans certaines ruches. Il est toutefois impossible d'établir une corrélation précise entre l'état du cadre et son activité. Le cadre le plus actif (ruche 536 , cadre $6,1^{\text {re }}$ hausse) contient $5,61 \mu \mathrm{Ci}$; il est très voisin de cadres très comparables par leur état de remplissage ( $\mathrm{n}^{\mathrm{os}} 4$ et 5 ) dont l'activité est faible $(0,51$ et $0,70 \mu \mathrm{Ci})$. Les cadres de la première hausse de la ruche 556 contiennent du miel operculé mais ont une radio-activité plus faible que celle de cadres équivalents de la ruche 571 .

Une auto-radiographie (Figure 3) réalisée sur un cadre dont on a par ailleurs la photographie (Figure 4) montre très nettement que le passage du sirop marqué vers les hausses conduit à une dispersion du produit dans toute la ruche avec toutefois des concentrations locales plus ou moins importantes.

Les données du tableau 4 peuvent être facilement transformées en pourcentages. En faisant le rapport hausse par hausse entre la radio-activité théori- 


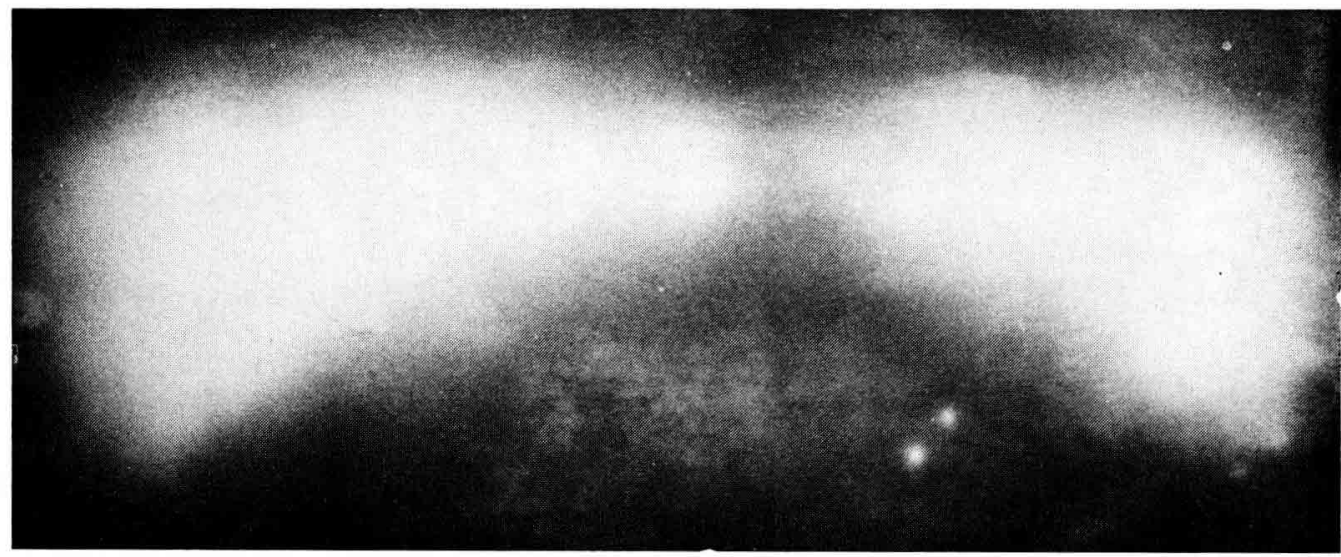

FIg. 3. - Auto-radiographie de contact oblenue avec un cadre de hausse (FIG. 4)

Fxposition : 20 jours.

Film Kodirex Kodak $30 \times 40 \mathrm{~cm}$.

Les zones blanches diffuses montrent la concentration de la radio-activité sur les parties operculées du cadre. cellules.

Deux points nettement séparés, à droite de l'image, mettent en évidence la radio-activité de deux

Une zone moins nette, milieu de l'image, fait apparaître des cellules contenant du pollen faiblement radio-actif du fait de son malaxage avec du sirop marqué.

Aвв. 3. - Konlakt-Röntgenaufnahme einer Wabe des Aufsatzes (Aвв. 4)

Film Kodirex Kodak $30 \times 40 \mathrm{~cm}$. Wabe.

Die diffus weissen Stellen zeigen die Konzentration der Radioaktivität in den verdeckelten Teilen der

Zwei deutlich abgegrenzte Punkte, rechts im Bild, zeigen die Radioaktivität zweier Zellen.

Eine weniger klare Stelle in der Bildmitte zeigt Pollenzellen die schwach radioaktiv sind, weil der Pollen mit markiertem Zuckerwasser verknetet wurde.

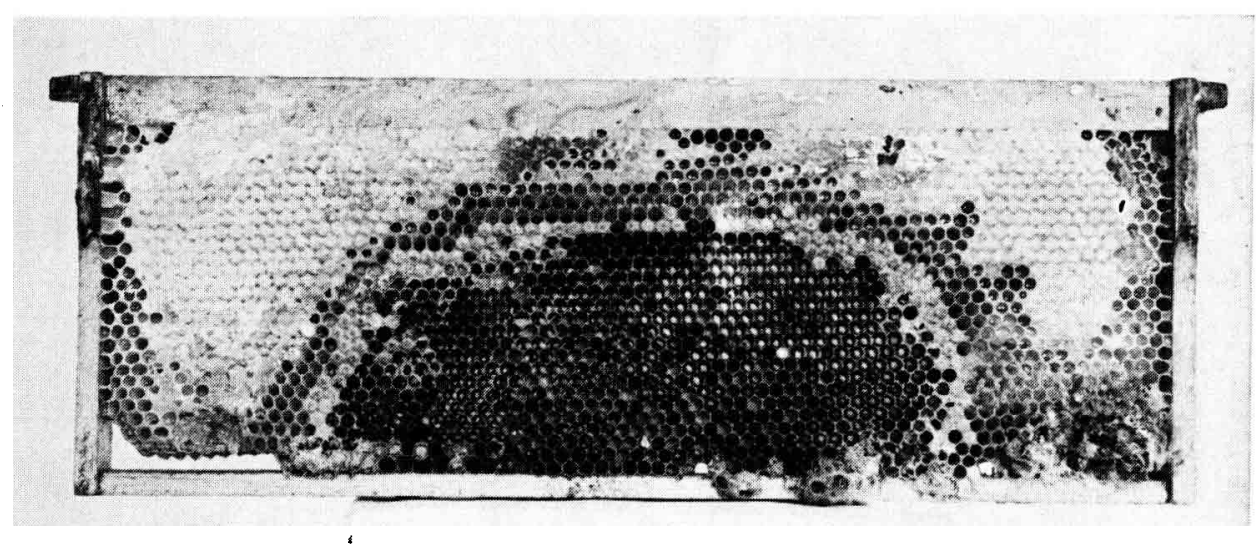

FIG. 4. - Cadre de hausse ayant permis d'obtenir l'auto-radiographie (FIG. 3)

Au-dessous des parties de miel operculé une ligne de couvain apparaît nettement. Au centre, des cellules contiennent du pollen.

Les autres cellules sont vides et le brillant de ces cellules est dû à un reflet.

Авв. 4.- Aufsatzwabe, von der die Röntgenaufnahme gemacht wurde (Авв. 3)

Unterhalb der Stellen mit verdeckeltem Honig ist eine Brutzeile deutlich zu erkennen. In der Mitte Pollenzellen.

Die anderen Zellen sind leer. Der Glanz ist eine Reflexerscheinung. 

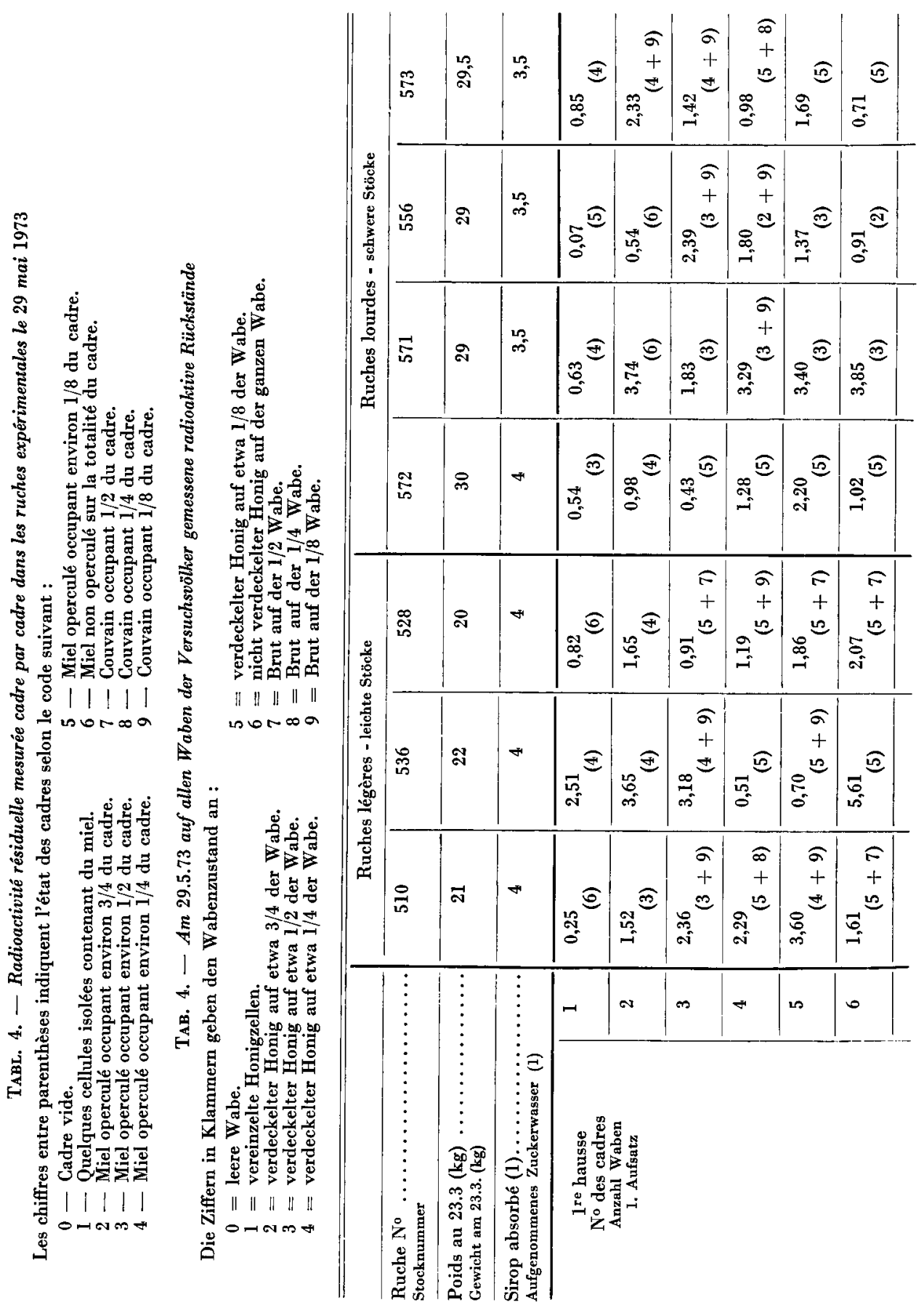
Étude du Stockage des réserves

119

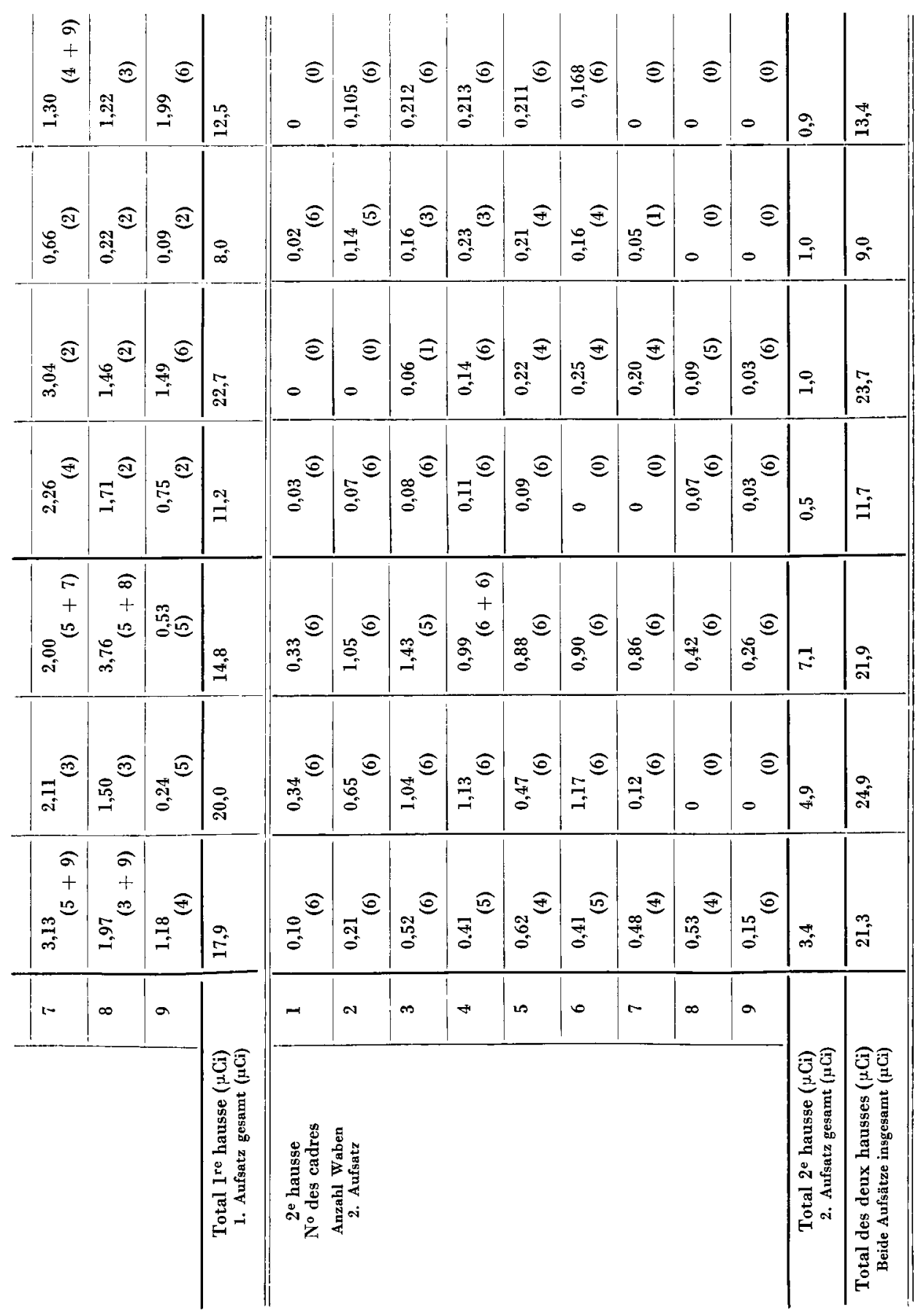


que totale possible au 29 mai et la radio-activité effectivement mesurée on peut établir le tableau 5 qui montre qu'au total, dans les conditions expérimentales que nous avons réunies, il peut rester dans les hausses au moment de la récolte de 2,4 à $6,3 \%$ du produit de marquage.

TABL. 5. - Radio-activité théorique restante et radio-activité mesurée le 29 mai 1973 (en \% de la radio-activité théorique restante)

TAB. 5. - Theoretisch verbleibende Radioaktivität und am 29.5 .73 gemessene Radioaktivität (in Prozenten der theoretisch verbleibenden Radioaktivität)

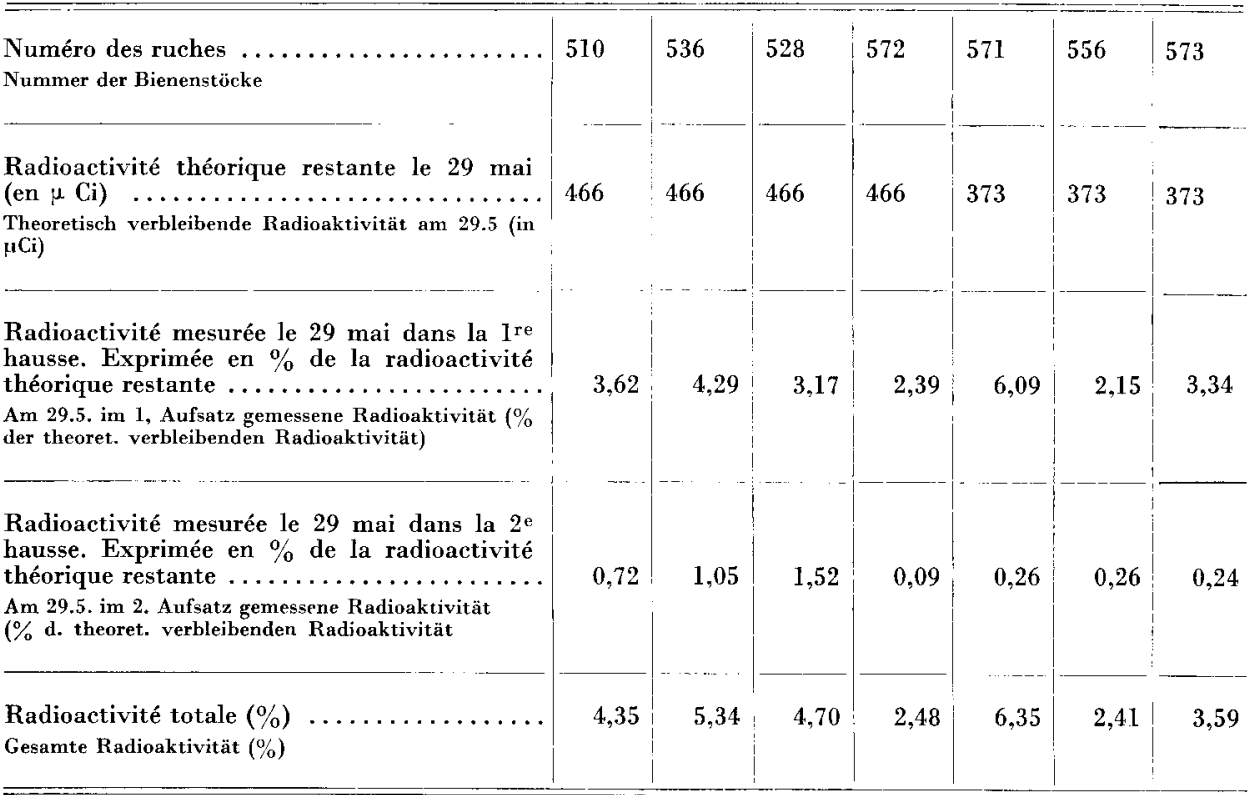

Cette donnée peut être convertie en sucre pur (Tableau 6). On aboutit alors à cette conclusion que dans le cas étudié et que nous avons voulu aussi près que possible des conditions de la pratique apicole, le miel récolté contient après un nourrissement stimulant de 4 litres de sirop à $600 \mathrm{~g}$ de sucre par litre, de $2,0 \mathrm{~g}$ à $8,4 \mathrm{~g}$ de sucre pur par $\mathrm{kg}$ de miel. Dans le cas où le sirop aurait contenu une drogue (sulfamide ou antibiotique) à la dose de $1 \mathrm{~g}$ par litre, ce qui correspond à l'usage courant, le miel récolté aurait contenu de 3,5 à $14 \mathrm{mg}$ de cette drogue par $\mathrm{kg}$ de miel, soit 3,5 à $14 \mathrm{ppm}$. Notons cependant qu'on ne dépasse pas normalement 2 g pour un traitement, et qu'on les administre dans un litre de sirop ce qui permet de ramener nos estimations à la moitié sinon au $1 / 4$ de leur valeur.

\section{DISCUSSION}

L'expérience de marquage d'un sirop de nourrissement que nous avons réalisée permet de tirer un certain nombre de conclusions. 
TABL. 6. - Influence da nourrissement sur la composition du miel

TAB. 6. - Einfluss der Fütterung auf die Zusammensetzung des Honigs

\begin{tabular}{|c|c|c|c|c|c|c|c|}
\hline $\begin{array}{l}\text { Numéro des ruches } \ldots \ldots \cdots \cdots \cdots \cdots \cdots \\
\text { Nummern der Bienenstöcke }\end{array}$ & 510 & 536 & 528 & 572 & 571 & 556 & 573 \\
\hline $\begin{array}{l}\text { Volume du sirop consommé (en l.) .......... } \\
\text { Menge des aufgenommenen Zuckerwassers (in } 1 \text { ) }\end{array}$ & 4 & 4 & 4 & 4 & 3,5 & 3,5 & 3,5 \\
\hline $\begin{array}{l}\text { Poids de la récolte de miel (en } \mathrm{kg}) \ldots \ldots \ldots \ldots \\
\text { Gewicht des geernteten IIonigs (in } \mathrm{kg} \text { ) }\end{array}$ & 24 & 19,3 & 13,4 & 15 & 20,5 & 22,9 & 18 \\
\hline $\begin{array}{l}\text { Poids du sucre de nourrissement en hausse } \\
\text { (exprimé en g par kg de miel) } \ldots \ldots \ldots \ldots \ldots \ldots \\
\text { Gewicht des Futterzuckers im Aufsatz (in g je kg Ho- } \\
\text { nig) }\end{array}$ & 4,2 & 6,5 & 8,4 & 3,8 & 6,2 & 2,0 & 4,0 \\
\hline $\begin{array}{l}\text { Poids du médicament ajouté au sirop de nour- } \\
\text { rissement (exprimé en mg par kg de miel)..... } \\
\text { Gewicht des dem Futterzuckerwasser zugesetzten } \\
\text { Medikaments (in mg je kg Honig) }\end{array}$ & 7,2 & 10,8 & 14 & 6,4 & 10,5 & 3,5 & 6,6 \\
\hline
\end{tabular}

On constate tout d'abord que, comme les nombreux travaux antérieurs, qu'il est super flu de citer ici, l'ont montré, la nourriture liquide, sirop de sucre ou nectar, qui entre dans la ruche subit de la part des abeilles un traitement complexe qui exige de multiples passages par le jabot et qui aboutit à une certaine homogénéisation physico-chimique. Le fait qu'un apport de sirop relativement minime laisse des traces dans tous les cadres des deux hausses, mises en place après la fin du nourrissement, est la preuve évidente des remaniements matériels constants dont la ruche est le siège.

L'ensemble des rayons constitue un milieu en perpétuelle évolution en fonction du développement ou de la régression du couvain ainsi que des apports alimentaires, nectar et pollen. L'extension du nid à couvain, en particulier, impose des déplacements de la nourriture et des modifications de son agencement. Dès la pose des hausses et leur occupation par les abeilles un courant ascendant de nourriture se produit et ce courant n'intéresse pas seulement le nectar fraîchement récolté mais aussi les provisions plus anciennes, probablement non encore operculées. Ce mouvement est général, c'est-à-dire qu'on constate son existence dans toutes les ruches, mais il est d'inégale importance selon les colonies.

La répartition dans les cadres de hausse du sirop marqué semble se faire au hasard car si on le retrouve partout on ne trouve cependant pas de gradient spatial de radio-activité clairement interprétable, bien que la géométrie de l'expension vers le haut de la ruche soit relativement régulière. Ceci prouve que le sirop marqué ne constitue qu'une partie des matières transportées et qu'il est 
pris au hasard par les ouvrières dans la masse liquide dont elles ont entrepris l'évacuation vers le haut. On peut, dans ce cas, comparer le travail des abeilles à celui d'une équipe d'aveugles chargés de placer des boîtes de conserves dans les rayons d'un magasin, les boîtes étant toutes semblables mais contenant des produits différents identifiables seulement par leur étiquette; bien que parfaitement rangées les boîtes ne pourraient pas être classées par nature du contenu.

L'importance du courant ascendant de nourriture liquide au moment où débute la miellée est probablement sujette à des variations sensibles en fonction de facteurs internes et externes : encombrement du corps de ruche, étendue du couvain, état des rayons latéraux, intensité de la miellée, conditions atmosphériques, etc. On peut également soupçonner l'influence d'un facteur génétique propre à la colonie car on sait que les caractéristiques de l'agencement interne de la ruche sont variables selon les races géographiques et, très certainement, selon les populations.

Le taux de transfert vers les hausses que nous avons mis en évidence est relativement faible puisqu'il reste, dans tous les cas, inférieur à $7 \%$ et qu'il est en moyenne de $4,17 \%$. On peut donc considérer que la grande masse du sirop marqué a été consommée ou stockée dans le corps de ruche. Des expériences complémentaires qui n'ont pas pu être mises en place en 1973 seraient nécessaires pour faire la part exacte de la consommation et du stockage.

La mise en évidence d'une façon précise des mouvements subis par le sirop marqué amène encore un certain nombre de remarques d'ordre théorique et pratique.

L'analyse pollinique permet très souvent de mettre en évidence dans le miel la présence de pollens dont la récolte est forcément antérieure à la pose des hausses. C'est le cas, par exemple, du pollen de saule (Salix sp.) provenant d'une miellée très précoce et qu'on retrouve régulièrement dans les miels récoltés en juin. Le miel de saule stocké dans le corps de ruche en mars-avril doit donc passer en partie dans les hausses tout comme notre sirop marqué. Certains décalages entre le spectre pollinique attendu et celui qu'on trouve effectivement à l'analyse trouvent donc une explication simple, jusqu'ici soupçonnée mais non démontrée.

La crainte des apiculteurs et des consommateurs de voir passer le sucre de nourrissement dans le miel apparaît comme fondée, du moins dans l'absolu. Le calcul a montré que, dans le cas précis de notre expérience, on peut estimer que le miel récolté est susceptible de contenir jusqu'à $8 \mathrm{~g}$ de sucre par kg et, en moyenne, $5 \mathrm{~g}$, soit 5 pour mille. Encore faut-il tenir compte du fait que ce sucre étant soumis à l'action de l'invertase ne se retrouve pas à l'état de saccharose mais sous la forme des sucres dominants du miel, glucose et lévulose. De toute façon, on peut affirmer, d'une part, que la quantité de sucre de nourrissement effectivement passée dans le miel dans de telles conditions n'est décelable par 
aucune méthode analytique connue et, d'autre part, qu'il ne peut en résulter aucun préjudice pour le consommateur.

Un point reste à discuter, celui de savoir si, aux doses effectivement retrouvées, un sirop médicamenteux aurait amené dans le miel récolté des quantités appréciables d'antibiotique ou de sulfamide. En nous plaçant dans les conditions expérimentales décrites on peut estimer à $14 \mathrm{mg}$ par $\mathrm{kg}$ de miel (14 $\mathrm{ppm}$ ) au maximum et à $8,4 \mathrm{mg}$ par $\mathrm{kg}$ en moyenne la quantité de substance active qui serait passée dans le miel. Dans les conditions normales de la pratique apicole, c'est-à-dire à raison de $2 \mathrm{~g}$ de substance active par ruche, on $n$ 'aurait pas dépassé 4 à 5 ppm ce qui paraît être au voisinage de la dose limite admissible de 6 ppm pour la terramycine (Chambonnaud, 1968). Encore faut-il tenir compte du fait que la consommation journalière du miel est faible et rarement supérieure à 20 à $30 \mathrm{~g}$. On sait que la posologie des antibiotiques et des sulfamides pour l'homme adulte se chiffre en g par jour (2 à 3 g par jour pour la streptomycine, par exemple). La consommation de 20 grammes de miel par jour apporterait, en moyenne, $0,08 \mathrm{mg}$ de substance active, soit à peu près 30000 fois moins. Il est permis de s'interroger sur l'importance physiologique de telles doses mais il ne nous appartient pas d'en juger.

Il est intéressant de confronter nos résultats à ceux qui ont été obtenus par Chambonna ud (1968) qui a dosé la sanclomycine dans des miels naturels de callune provenant de ruches traitées antérieurement. Cet auteur a retrouvé, au maximum 2 ppm de sanclomycine et, le plus souvent, des doses inférieures à 1 ppm. Bien que les conditions expérimentales ne soient pas exposées de façon détaillée on peut considérer que ces résultats sont en accord avec les nôtres qui ne tiennent pas compte de la destruction progressive des antibiotiques dans le miel qui a pu jouer un rôle important dans le cas des expériences de ChambonNAUD, le miel de callune étant récolté en octobre. On notera encore que Chambonnaud estime qu'il ne semble pas qu'une telle quantité d'antibiotique puisse présenter un danger quelconque pour le consommateur.

L'expérience que nous avons faite se place dans un contexte apicole bien précis, à savoir l'utilisation de ruches verticales, dans la région parisienne. Nous ne pouvons pas extrapoler à l'utilisation de ruches plus petites ou plus grandes ou dont l'agrandissement se fait progressivement dans le sens horizontal (ruches allemandes par exemple). Nous ne pouvons pas non plus donner des précisions sur ce qui se serait passé en cas de miellée nulle, très faible ou très forte.

Si nous nous en rapportons à des informations verbales ou à des articles tels que celui de J. Marshall (1970) paru dans la presse apicole, il est vraisemblable que, de façon accidentelle, des remontées importantes de sirop de sucre dans les hausses peuvent se produire sans qu'on en connaisse exactement les causes. Dans de tels cas l'utilisation de sucre dénaturé constitue un moyen satis- 
faisant pour alerter l'apiculteur alors que le sucre ordinaire ne laisse pas de traces directement visibles.

La méthode que nous avons mise au point, en raison de sa très grande sensibilité et de l'abondance des données chiffrées qu'elle procure, nous semble bien adaptée aux recherches sur le nourrissement des abeilles et sur la nutrition des colonies; c'est pourquoi nous envisageons de reprendre ultérieurement nos essais en faisant varier divers paramètres de façon à connaître les conséquences de situations autres que celles que nous avons pu étudier au cours d'une unique campagne.

Reçu pour publication en novembre 1973.

Eingegangen im November 1973.

\section{ZUSAMMENFASSUNG}

Die Verwendung von Radio-Isotopen zur Markierung von Zuckerwasser, das an Bienen verfüttert wird, ermöglichte schon die Durchführung zahlreicher Versuche, deren Hauptziel die Erforschung der verschiedenen Ernährungsformen war. Es erschien vielversprechend, die gleiche Methode anzuwenden, doch nicht mehr im Laboratorium, um Bienen zu markieren sondern in der Natur, um die Bewegungen zu untersuchen, denen die Futterreserven im Innern des Bienenstockes unterworfen sind. Die Beobachtungen der Imker und Versuche mit gefärbtem Zuckerwasser haben ergeben, dass Fütterungszucker sich sehr wohl in mehr oder minder bedeutenden Mengen im Honig wiederfinden kann. Ausserdem haben andere Versuche gezeigt, dass Medikamente, die den Bienen zur Krankheitsbekämpfung verabreicht werden, unter Umständen ebenfalls im Honig nachgewiesen werden können. Diese Versuche wurden jedoch, ganz allgemein gesprochen, bisher nicht in einer der derzeitigen Imkerpraxis entsprechenden Weise durchgeführt. Deshalb haben wir versucht, Bedingungen zu schaffen, die der Praxis möglichst nahekommen, das Material zu verwenden, das z.Zt. in der Pariser Gegend üblich ist und die Fütterung so durchzuführen, wie sie von den meisten Bienenzüchtern gehandhabt wird.

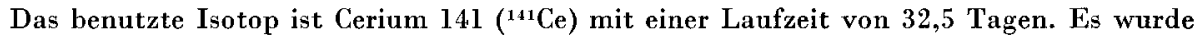
dem Zuckerwasser im Verhältnis von $500 \mu \mathrm{Ci}$ je Liter beigemischt. Im Laboratorium wurde nachgewiesen, dass die angewandte Dosis Cerium für die Bienen nicht giftig ist. Eine radiochemische Wirkung ist von vornherein ausgeschlossen, da die Bienen gegen radio-aktive Strahlen ausserordentlich resistent sind. Zur Verhütung einer gefährlichen Verseuchung der Umwelt wurden alle notwendigen Vorsichtsmassnahmen getroffen.

Sieben Bienenvölker der allgemein üblichen schwarzen Rasse in 10-Waben-Dadantstöcken erchielten zwischen dem 23. März und 13. April wöchentlich je ein Liter markiertes Zuckerwasser. Es wurden zwei Gruppen gebildet, von denen die eine aus Völkern mit normalen Vorräten bestand, während die Vorräte der zweiten Gruppe so gehalten waren, dass eine Fütterung notwendig war (Tab. 1).

Die Volksstärke war in allen Völkern der Jahreszeit entsprechend normal. Alle Königinnen waren Geschwister und 1972 gezogen worden. Am 19. April, nach dem Einsetzen der Rapstracht, wurden die Aufsätze gegeben und am 29. Mai, nach Beendigung der Tracht, wieder abgenommen.

Zum Messen der radioaktiven Rückstände in den Aufsätzen wurde eine Spezialvorrichtung benutzt, die zehn Messungen je Wabe ermöglichte. (Abb. $1+2)$ 


\section{Ergebnisse}

Da die Witterungsverhältnisse günstig waren, gab es eine gute Honigernte (Tab. $2+3$ ). In der Zeit nach dem Aufsetzen der Honigräume betrug die Gewichtszunahme 12 bis $23 \mathrm{~kg}$ je Volk.

Die Messung der radioaktiven Rückstände auf allen Waben zeigte, dass sich das markierte Zuckerwasser in allen Waben aller Völker wiederfand und zwar dem Honigvorrat entsprechend, ohne jedoch gleichmässig verteilt zu sein. Benachbarte, gleich schwere Waben können eine recht unterschiedliche Radioaktivität aufweisen. Zwischen den beiden Gruppen von Bienenvölkern gab es keinen bemerkenswerten Unterschied. Das Röntgenbild einer Wabe (Abb. $3+4$ ) zeigt die Verteilung von ${ }^{14} \mathrm{Ce}$ sehr deutlich.

Wenn man die aus dem Fütterungs-Zuckerwasser stammende Saccharosemenge berechnet, die in den Honig gelangte, erhält man Werte zwischen 2 und 8,4 g je kg Honig.

\section{Diskussion}

Die durchgeführten Versuche bestätigen die Tatsache, dass die Bienen ihre flüssigen Futtervorräte umtragen können. Zur Zeit, wo die Aufsätze gegeben werden, zeichnet sich eine aufsteigende Bewegung ab, die vermutlich mit der Ausdehnung des Brutnestes zusammenhängt.

In der praktischen Bienenzucht sind die in den Honig eingebrachten Zuckermengen zu gering, um durch die Analyse festgestellt zu werden. Die manchmal von Imkern festgestellte bedeutende Menge von Fütterungszucker im Honig ist vermutlich auf besondere Umstände zurückzuführen, deren Aufhellung andere Nachforschungen notwendig macht.

In bezug auf das Eindringen medikamentöser Substanzen in den Honig muss man sehr vorsichtig sein und darf nicht vergessen, dass der Honig absolut frei von solchen Substanzen bleiben muss. Die Berechnung zeigt, dass bei den Gep flogenheiten der derzeitigen Imkerpraxis Mengen von 4 bis $5 \mathrm{mg}$ in $1 \mathrm{~kg}$ Honig gelangen können. (Tab. 6)

Neue Versuche mit der gleichen Technik sind noch notwendig, um den Ein fluss gewisser Faktoren wie Zeitpunkt und Menge des Trachtangebotes, Stärke des Bienenvolkes, Witterungsverhältnisse usw. zu untersuchen.

\section{RÉFÉRENCES BIBLIOGRAPHIQUES}

Chambonnaud J.-P., 1968. - Contribution à la recherche des antibiotiques dans le miel. Bull. apic. Doc. sci. tech., 9 (1), 133-197.

Courtois G., Lecomte J., 1958. - Sur un procédé de marquage des abeilles butineuses au moyen d'un radio-isotope. C. $R$. Acad. Sci., 247, 147-149.

Courtors G., Lecomte J., SAlleron F., 1961. - Étude des échanges de nourriture à l'intérieur de la ruche entre les abeilles ouvrières (Apis mellifica L.). C. R. Acad. Sci., 252, 10571059.

Dounult Ph., 1967. - In fluence du nid à couvain sur les échanges de nourriture entre ouvrières d'abeilles (Apis mellifica L.). C. R. Acad. Sci., D, 264, 1092-1095.

Gösswald K., Kloft W., 1958. - Radioaktive Isotope zur Erforschung des Staatenlebens der Insekten. Umschau, 24, 743-745.

HadorN H., ZürChER K., 1963. - Über Zuckerfütterungshonig. Mitt. Geb. Lebensmittelunters. Hyg., 54. (4), 322-330. 
Jachimowicz Th., 1964. - Der Ein fluss der Trockenzuckerfütterung auf die Honigqualität. Bodenkultur, 15 (3), 285-299.

Marsharl J., 1970. - How pure is your honey? Br. Bee J., 21 March, 63-64.

Nixon H. L., Ribbands C. R., 1952. - Food transmission within the honeybee community. Proc. r. Soc., B, 140, 43-50.

Racoveanu N., Popa Al., Tarcitu E., 1965. - La Radioactivité du miel d'abeilles dans la région de Bucarest. Bull. apic. Doc. sci. tech., 8, 147-151.

Rousseau M., Tabarly 0., 1962. - L'emploi des antibiotiques en apiculture : leur action dans la ruche, leur présence dans le miel. Bull. apic. Doc. sci. tech., 5 (2), 155-176.

Vorworl G., 1964. - Die Messung der elektrischen Leitfähigkeit des Honigs und die Verwendung der Messwerte zur Sortendiagnose und zum Nachweis von Verfälschungen mit Zuckerfütterungshonig. $Z$. Bienenforsch., 7 (2), 37-47. 